\title{
Viscoelastic deformation of articular cartilage during impact loading
}

\author{
Lorna Edelsten, Janet E. Jeffrey, Leanne V. Burgin ${ }^{1}$, Richard M. Aspden
}

Bone and Musculoskeletal Research Programme, Division of Applied Medicine, University of Aberdeen, Institute of Medical Sciences, Foresterhill, Aberdeen AB25 2ZD, UK

${ }^{1}$ Current address: Enterprise \& Innovation Office, University of Leeds, Charles Thackrah Building, 101 Clarendon Road, Leeds LS2 9LJ, UK

Address for correspondence

Professor R.M. Aspden

Division of Applied Medicine,

University of Aberdeen,

Institute of Medical Sciences,

Foresterhill,

Aberdeen AB25 2ZD,

Scotland, UK

Tel: +44 1224552767

Fax : +44 1224559533

e-mail: r.aspden@abdn.ac.uk 


\section{Summary}

Articular cartilage is a highly hydrated fibre composite material that provides a resilient, lowfriction bearing surface covering bones where they articulate. The literature suggests that the tissue becomes increasingly elastic, less viscoelastic, as the loading rate increases, i.e. that hysteresis, the energy lost between loading and unloading, will decrease with increasing strain-rate. Here we show, using a controlled impact, that hysteresis increases with strain rate. No fluid was lost during the deformation and the ratio of the radial to the axial strains, Poisson's ratio, measured using highspeed video recording, increased as the tissue was deformed, starting close to zero and tending towards that for an isovolumetric deformation. The decreasing coefficient of restitution, a measure of the hysteresis, was modelled using a non-linear viscoelastic element, as a first approximation. These results indicate that the tissue remains viscoelastic with increasing strain rate, dissipating energy which might otherwise generate cracks in the matrix.

\section{Keywords}

articular cartilage; viscoelasticity; mechanical properties; impact loading; Poisson's ratio 


\section{Introduction}

Articular cartilage is a fibre-composite biomaterial that provides a low-friction bearing surface to bones where they articulate in synovial joints. Trauma may result in a joint being subjected to extreme forces applied very rapidly, for example, due to a car accident or sports injury. In these cases, the cartilage is subjected to an impact load and transmits the shock to the underlying bone. ${ }^{1 ; 2}$ Such trauma may lead to cartilage degeneration and commonly results in secondary osteoarthritis (OA); the most common musculoskeletal disease characterised by the breakdown and eventual loss of cartilage, chronic pain and severe disability. The precise mechanisms linking trauma-induced joint injury to the subsequent onset of $\mathrm{OA}$ are still unclear but changes in the articular cartilage immediately after trauma are likely to be important factors in the progression of the disease..$^{3 ; 4}$

The complexity of the tissue structure and the non-linear mechanical properties mean that developing materials for cartilage repair, using either synthetic analogues or tissue engineered implants, presents a huge challenge. The cartilage matrix comprises collagen, a rope-like fibrous protein, that is arranged ${ }^{5}$ to provide reinforcing to a proteoglycan gel which, because of its high fixed-charge density, is highly hydrated..$^{6-9}$ The water content of the tissue is about $70-80 \%(\mathrm{v} / \mathrm{v})$. Slowly-applied loads displace the fluid and frictional drag arising from fluid flow past the glycosaminoglycan chains has been used to explain the remarkably low permeability of the tissue. ${ }^{10}$ Fluid flow through a poroelastic solid matrix commonly provides the basis for models of the tissue. $^{11-14}$ Since the early elastic models, ${ }^{15}$ cartilage has been characterised as viscoelastic, ${ }^{16}$ biphasic $^{17}$ or triphasic. ${ }^{18}$ Other descriptions have used Biot's consolidation theories. ${ }^{19}$ The most common model is based on biphasic theory and this has been extended to include poroviscoelasticity, ${ }^{20 ; 21}$ transverse isotropy ${ }^{22}$ and fibril reinforced poroelasticity. ${ }^{23 ; 24}$ More recent refinements include tension-compression and strain non-linearities ${ }^{25}$ and swelling. ${ }^{26}$ None of these models contains a provision for impact loading. 
A key difficulty facing tissue engineering of cartilage is matching the mechanical properties of the replacement material with that of the natural tissue over several orders of magnitude of loading rates experienced during daily activities. Because articular cartilage is viscoelastic its behaviour is time-dependant; it exhibits a non-linear stress-strain relationship that depends on the rate of loading. Whereas there are many studies of cartilage subjected to low rates of loading, typically strain rates of $<1 \mathrm{~s}^{-1}$, there are fewer at high rates of loading, strain rates $\sim 10-1000 \mathrm{~s}^{-1}$. At these high strainrates, inertia precludes significant water movement and it is commonly assumed that the tissue becomes more elastic, ${ }^{27 ; 28}$ i.e. having a smaller time-dependency, and lower hysteresis. Hysteresis means that the stress-strain path during unloading does not follow that developed during loading. The area under the loading curve is a measure of the energy stored in the deformation and that under the unloading curve the energy returned. The ratio of the energy returned to that stored is described by the energetic coefficient of restitution, which can, therefore, vary between one for perfectly elastic loading to zero if all the energy is dissipated. Values for articular cartilage are unknown.

The Young's modulus and Poisson's ratios describe the response of a material to deformation. Although originally defined for small deformations of elastic materials they can be adapted to describe large deformations. At slow rates of loading the measured Young's modulus of cartilage is typically quoted as approximately 1-10 $\mathrm{MPa} .^{29-31}$ The stress-strain relationship, however, is not linear and values at high rates of loading depend on the stress as well as on the rate of loading. Maximum moduli, calculated at the steepest part of the stress-strain curve, are reported in the range up to about $200 \mathrm{MPa} .{ }^{32-35}$ At large strain rates, however, the modulus has been reported to lose much of its dependency on strain rate. ${ }^{28 ; 35}$ In an isotropic material Poisson's ratio is the negative ratio of transverse to applied longitudinal strain. It has been measured in cartilage subjected to slow rates of compression and estimates vary from almost zero to 0.5 depending upon the approach adopted (Table 1). Similar values have been calculated using biphasic theory in which one of the 
variables is $v_{\mathrm{s}}$, the Poisson's ratio of the solid matrix through which fluid flows. ${ }^{13}$ Although it is commonly assumed that Poisson's ratio has to be less than 0.5 , this is only true for isotropic, homogenous materials subjected to small strains. For many modelling purposes it has been assumed that cartilage is isotropic and incompressible, i.e. experiencing no volume change, resulting in a value for $v_{\mathrm{s}}$ of $0.5 .^{36}$ There appears to have been no previous attempt to measure Poisson's ratio in cartilage undergoing large strain deformations at high rates of loading.

In this study, we try to address some of these gaps in our understanding and report preliminary measurements of Poisson's ratio as a function of longitudinal strain in samples of articular cartilage subjected to an impact load and examine the role of hysteresis and the energetic coefficient of restitution. The hypothesis being tested is that cartilage behaves more elastically as strain-rate increases. We also present a simple theoretical analysis to approximate an impact on a viscoelastic material. The data presented and the modelling indicate that articular cartilage behaves like a nonlinear viscoelastic material at high strain rates.

\section{Experimental details}

Two experiments were performed: high-speed video recording of impact loading on human cartilage to measure tissue deformation and a series of impact loadings on bovine cartilage to measure the energetic coefficient of restitution. For the high speed video study, articular cartilage was obtained from femoral heads of two patients (both aged 85, one male, one female) undergoing hemiarthroplasty following a fractured neck of femur attributed to osteoporosis. Local Ethics Committee approval was obtained for the use of this surgical material which would otherwise have been discarded. The articular cartilage showed no evidence of fibrillation. Nine, full-depth cartilage samples, $5 \mathrm{~mm}$ in diameter, were removed from sites over the femoral head using a cork borer and 
scalpel $^{37}$ and stored in phosphate-buffered saline (PBS) to maintain hydration until testing. Just prior to testing, each sample was gently dried using damp gauze to remove surface water. Data for the coefficients of restitution were obtained from young bovine cartilage used as part of a previous study in which 83 samples of $5 \mathrm{~mm}$ diameter from the medial metacarpal joint surface were tested. ${ }^{35}$ All testing was done on the same day as tissue was removed from the joint.

An instrumented drop tower was used to apply a single defined impact to each articular cartilage sample. The energy and speed of impact were determined by the mass and drop height of the impactor. ${ }^{3 ; 38}$ The deceleration of the impactor and the force applied were measured at $50 \mathrm{kHz}$ by an accelerometer attached to the impactor and a force transducer underneath the sample, respectively. Force data were converted to engineering stress, $\sigma$, by dividing by the original cross-sectional area of the sample. Accelerometer data were integrated twice, to find the displacement, and divided by the original thickness of the sample to determine the engineering strain, $\varepsilon$. The mean strain rate was calculated from the maximum strain divided by the duration of the impact to that point. A full description of the data collection and analysis methods have been presented elsewhere. ${ }^{38}$

For high-speed video recording, six samples (3 from each patient) were tested at each drop height of 25, 50 and $75 \mathrm{~mm}$ using a $500 \mathrm{~g}$ impactor. A Photo-Sonics Phantom V7 high speed video system (EPSRC Engineering Instrument Pool, Rutherford Appleton Laboratory, Oxfordshire) was used to record the deformation of the cartilage during impact. The impacts were recorded at 10,600 frames per second with a resolution of $512 \times 384$ square pixels. Before recording, the vertical and horizontal distances were calibrated by recording images of a ruler placed where the specimen would be. The camera was focused on this plane and not subsequently adjusted once the ruler was removed and replaced by a specimen. After recording, images were transferred to a PC for analysis using Image Pro Plus (version 5.0, Media Cybernetics, Wokingham Berkshire, UK). The measurement tool within the software was calibrated vertically and horizontally on the images of 
the ruler. These calibrations, as expected, always produced the same factor for both directions. The distance of the camera from the object and the depth of field were such that the front and the middle of the sample were in focus. Measurements of the height and width of each sample were made from each frame (Fig. 1). The height was measured at three sites because the front of the loading platen was not in focus and consequently the top and bottom edges of the sample were not always clear. The longitudinal, $\varepsilon_{z}$, and radial, $\varepsilon_{r}$, strains were determined from the change in dimension divided by the initial dimension. Poisson's ratio, $v$, was calculated at each time point from $v=-\varepsilon_{r} / \varepsilon_{z}$.

From each of the bovine cartilage samples a force-displacement curve was produced for loading and unloading phases as described previously. ${ }^{35}$ Unloading was the unconstrained rebound of the impactor. The impactor mass was $100 \mathrm{~g}$. Drop heights used and the number of samples tested, $N$, were $25 \mathrm{~mm}(N=21), 50 \mathrm{~mm}(N=23), 80 \mathrm{~mm}(N=20)$ and $100 \mathrm{~mm}(N=19)$. The energy of deformation, $W_{c}$, was found from the area under the loading curve by integrating to the maximum strain (Origin Software, Version 6.1; Aston Scientific Ltd, Stoke Mandeville), and the energy released during restitution, $W_{r}$, by integrating the unloading curve from this point back to zero strain. The square of the energetic coefficient of restitution is then given by $e_{*}^{2}=-W_{r} / W_{c}{ }^{39}$ For a perfectly elastic collision $e_{*}=1$, and for a plastic collision, in which all the kinetic energy is dissipated, $e_{*}=0$. Least squares curve fitting was done using SigmaPlot (v.10, Systat Software Inc.).

\section{Results and discussion}

\section{Poisson's ratio}

Articular cartilage deforms non-linearly when subjected to an impact load and a typical impact is shown in the supplementary video. Stress and strain data recorded from adjacent sites in the same femoral head for three different drop heights of an impactor are shown in Fig. 2 and it can be seen that the peak stress does not coincide with the peak strain. The initial parts of the loading curves lie 
very close together but the peak strain increases as the drop height is increased. The mean values of the maximum longitudinal strain, radial strain, and Poisson's ratio calculated from the high-speed video images are shown in Table 2 for each drop height, along with the mean strain rate. Strain rates were considerably greater for samples from femoral head B because the mean thickness of the cartilage over the femoral head was $1.65(2.4) \mathrm{mm}$ compared with $2.30(0.12) \mathrm{mm}$ for samples from femoral head A. Longitudinal strain values calculated from the accelerometer are also shown in Table 2 and were almost identical to those measured from the images. The variation of Poisson's ratio with applied strain for each drop height is shown in Fig. 3. There was considerable variability but values typically increased from close to zero and, at large strains, approximated the theoretical value for an isovolumetric deformation calculated from

$$
v=\frac{1}{\varepsilon_{z}}\left(1-\sqrt{\frac{1}{1+\varepsilon_{z}}}\right)
$$

Where $\varepsilon_{z}$ is the engineering strain (deformation / original dimension) in the axial direction. Engineering strains were used in preference to logarithmic strains to enable comparison with previous studies. Using logarithmic, or Hencky, strain $\left(e_{t}=\ln \left(x_{i} / x_{0}\right)\right)$, where $x_{i}$ and $x_{0}$ are the deformed and original dimensions respectively) results in Poisson's ratio being constant and equal to 0.5 for all applied strains for an isovolumetric deformation. Graphs showing Poisson's ratio calculated using logarithmic strains are shown in the Supplementary information. Having only one camera meant that anisotropic deformations could not be recorded and this, together with the bulging of the edge of the sample, precluded the calculation of accurate volume changes and anisotropic Poisson's ratios that will be addressed in future studies.

The initially low values of Poisson's ratio indicate that the volume of the tissue is not preserved during the deformation but may be close to that expected for constant volume towards the end of the loading phase as the loading rate falls to zero. Despite the high water content of cartilage no water was seen to be ejected from the sample during testing. Care was taken to remove only surface 
water before testing using a moistened gauze. ${ }^{40}$ In a previous study of bovine tissue we weighed the samples before and after testing and found no measurable mass loss within the resolution of the balance $(0.01 \mathrm{mg}$, sample mass $\sim 20 \mathrm{mg}) .{ }^{35}$ Despite the large deformations, axial compressive strains of the order of $50 \%$, these data support there being no bulk flow of water through the matrix during the test.

\section{Hysteresis}

Hysteresis was large and the area between the loading and unloading parts of the curves increased with impact velocity, although much of this was due to the increase in maximum strain due to the unconstrained impact. A decreasing value for the coefficient of restitution, however, indicated that hysteresis was actually increasing. The area under the unloading curve is the energy returned to the impactor by the rebound and the imaging shows that contact was maintained between the sample and the impactor throughout the unloading phase. These results show that the increasingly elastic behaviour of cartilage anticipated from the literature, ${ }^{2728}$ which would result in a reduction in hysteresis, does not occur. Testing was done in unconfined compression whereas in vivo the surrounding tissue will afford considerable support to the loaded region and restrict lateral deformation. This will make the tissue appear stiffer and it may make it appear more elastic. ${ }^{41 ; 42}$ This effect remains to be tested in future studies.

\section{Coefficient of restitution}

The coefficient of restitution data from bovine cartilage have been presented in a different form elsewhere ${ }^{35}$ but mean values are shown here plotted as a function of impact velocity $u_{0}$ (Fig 4 ). The coefficient of restitution, $e_{*}$, decreases with increasing impact velocity. To investigate the effects of an impact load on a viscoelastic material, Stronge ${ }^{39}$ has presented an analysis of an impact on a Maxwell element, i.e. a spring and dashpot in series. The Maxwell element is the simplest linear 
viscoelastic model and generates a restoring force that increases smoothly with compression. In addition, some kinetic energy of normal relative motion is restored during restitution. The coefficient of restitution, however, does not depend on the impact velocity. Introducing a non-linear dashpot in which the damping force depends not only on the velocity but also on the displacement, is one way in which this limitation can be overcome. Combining a non-linear dashpot in parallel with a spring produces a variation on a Kelvin-Voigt model for viscoelasticity in which the coefficient of restitution depends on impact velocity ${ }^{39}$ (Fig. 5).

A Kelvin-Voigt element comprises a spring, which is totally elastic and has a spring constant $k$, relating applied force, $F$, to displacement, $x$, so that $F=-k x$, and a dashpot. If the dashpot resists an applied force with a resistance proportional both to velocity and displacement then this may be written as $F=-c x \dot{x}$ where the damping coefficient is $c$. Arguably, there could be separate coefficients for the displacement and velocity terms but all that is required here is a way to describe the data, so the simplest description has been chosen and no effort is made to represent specific mechanisms within the tissue. Consider the contact of the impactor on the sample as a collision between two impacting masses, $m_{1}$ and $m_{2}$. If $z$ is the instantaneous distance between them, then the normal force, $F$, is the sum of the reactions of the spring and the dashpot and is given by

$$
F=-k z-c|z| \dot{z}
$$

The equation of relative motion is a second order differential equation given by

$$
M \ddot{z}-c z \dot{z}+k z=0
$$

where $M$ is the effective mass, $M^{-1}=m_{1}{ }^{-1}+m_{2}{ }^{-1}$. In Equation 2, the absolute sign has been omitted provided the initial condition $\dot{z}(0)=-u_{0}$ is the velocity at impact which is taken to be negative and $z$ is negative with $z(0)=0$. This is similar to the equation for damped harmonic motion and, although the nonlinear term makes the a solution more complicated, an outline of the derivation is 
given by Stronge. ${ }^{39}$ The coefficient of restitution, $e_{*}$, decreases with increasing impact velocity and if a non-dimensional relative velocity is defined by

$$
V \equiv c \frac{\dot{z}}{k}
$$

then an approximation to the relationship is given by

$$
e_{*} \approx\left(-V_{0}+e^{0.4 V_{0}}\right)^{-1}
$$

where $V_{0}=V(0)=-c u_{0} / k^{39}$. The fitted curve was derived from equation 4 and constrained to pass through $(0,1)$. This returned a value for $c / k=1.402$ (standard error $\left.0.092, R^{2}=0.87\right)$.

Simple analytical models of materials using the concepts of springs and dashpots are phenomenological and cannot be related easily to the composition and structure of the material. They can, however, provide an insight into the viscoelastic nature of the deformation, and clearly show, in this instance, that the tissue behaves as a non-linear viscoelastic material. The timedependent behaviour of cartilage during impact probably arises from mechanisms very different from those normally considered. Creep and stress relaxation, which have a long time-constant, arise largely from movement of water through the proteoglycan gel and this is traditionally modelled using poroelastic and multiphasic approaches as described above. During impact, when inertia will limit fluid flow, fibre reorientation and motion within the gel matrix are likely to occur in order to achieve the large strains observed. These will have much shorter time-constants and lend themselves to the descriptions used here; it is a matter of finding the simplest model that adequately describes the behaviour. In this case an impact on a linear Maxwell element would yield a coefficient of restitution that is independent of the impact velocity and this does not accord with the data. Introducing a non-linear element produces a coefficient of restitution that has similar behaviour to that shown here by cartilage, i.e. decreasing with impact velocity. ${ }^{39}$ The model may be too simple but as a first approximation it provides a reasonable fit to the existing data using just one 
adjustable parameter. The significance of the ratio of $c / k$ being 1.4 is not clear but the value indicates that elastic (represented by $k$ ) and dissipative forces (represented by $c$ ) are of similar magnitudes. This is not attempting to explain the behaviour of cartilage but provides a convenient and effective way of describing it.

\section{Conclusions}

The results of this preliminary study show that under high speed loading cartilage behaves as a nonlinear viscoelastic material, disproving the hypothesis of increasingly elastic behaviour. Poisson's ratio measurements suggest a loss of volume during loading, regained towards the end of the loading phase, and hysteresis increases with loading rate. Increasing hysteresis may provide a mechanism to enable energy to be dissipated instead of creating fracture surfaces, thereby limiting potential damage. Despite the limitations noted above, these results have implications for modelling the tissue where assumptions of small strains, constant moduli and Poisson's ratios that are constant and assume no volume change are often used. Measurements using more sophisticated imaging, and theoretical modelling are needed to enable these properties to be determined in more detail. Under high speed loading conditions, however, cartilage behaves like a viscoelastic material showing hysteresis and non-linear, time-dependent properties.

\section{Acknowledgments}

We thank the Orthopaedic surgeons of NHS Grampian for kindly letting us use tissue donated by their patients. We also thank the EPSRC loan pool for the loan of the high speed video equipment and Adrian Walker and Keith Hastings for kindly coming all the way to Aberdeen to teach us how to use it. LVB and JEJ were supported by Arthritis Research UK (formerly the Arthritis Research Campaign) on grant numbers A0542 and 16300 respectively. 


\section{Conflict of interest}

The authors have no conflicts of interest 


\section{References}

1. P. J. Atkinson and R. C. Haut, Journal of Orthopaedic Research, 1995, 13, 936-944.

2. J. B. Finlay and R. U. Repo, Medical and Biological Engineering and Computing, 1979, 17, 397403.

3. J. E. Jeffrey, D. W. Gregory, and R. M. Aspden, Archives of Biochemistry \& Biophysics, 1995, 322, 87-96.

4. J. E. Jeffrey, L. A. Thompson, and R. M. Aspden, Biochimica et Biophysica Acta, 1997, 1334, 223-232.

5. R. M. Aspden and D. W. L. Hukins, Proceedings of the Royal Society, 1981, B212, 299-304.

6. R. M. Aspden and D. W. L. Hukins, Matrix, 1989, 9, 486-488.

7. R. M. Aspden, Proceedings of the Royal Society, 1994, B-258, 195-200.

8. K. L. Goh, J. R. Meakin, R. M. Aspden, and D. W. Hukins, Journal of Theoretical Biology, 2007, 245, 305-311.

9. D. W. L. Hukins and R. M. Aspden, Trends in Biochemical Sciences, 1985, 10, 260-264.

10. I. S. Kovach, Biophys.Chem., 1996, 59, 61-73.

11. F. Boschetti, F. Gervaso, G. Pennati, G. M. Peretti, P. Vena, and G. Dubini, Biorheology, 2006, 43, 235-247.

12. V. C. Mow, M. C. Gibbs, W. M. Lai, W. B. Zhu, and K. A. Athanasiou, Journal of Biomechanics, 1989, 22, 853-861.

13. A. F. Mak, W. M. Lai, and V. C. Mow, Journal of Biomechanics, 1987, 20, 703-714.

14. A. Oloyede and N. D. Broom, Connective Tissue Research, 1994, 31, 75-86.

15. W. C. Hayes, L. M. Keer, G. Herrmann, and L. F. Mockros, Journal of Biomechanics, 1972, 5, 541-551.

16. J. R. Parsons and J. Black, Journal of Biomechanics, 1977, 10, 21-29.

17. V. C. Mow, S. C. Kuei, W. M. Lai, and C. G. Armstrong, Journal of Biomechanical Engineering, 1980, 102, 73-84.

18. W. M. Lai, J. S. Hou, and V. C. Mow, Journal of Biomechanical Engineering, 1991, 113, 245258.

19. A. Oloyede and N. D. Broom, Clinical Biomechanics, 1991, 6, 206-212.

20. A. F. Mak, J Biomech.Eng, 1986, 108, 123-130.

21. A. F. Mak, Biorheology, 1986, 23, 371-383. 
22. B. Cohen, W. M. Lai, and V. C. Mow, Journal of Biomechanical Engineering, 1998, 120, 491496.

23. L. P. Li, J. Soulhat, M. D. Buschmann, and A. Shirazi-Adl, Clinical Biomechanics, 1999, 14, 673682.

24. J. Soulhat, M. D. Buschmann, and A. Shirazi-Adl, Journal of Biomechanical Engineering, 1999, 121, 340-347.

25. F. Boschetti, G. Pennati, F. Gervaso, G. M. Peretti, and G. Dubini, Biorheology, 2004, 41, 159166.

26. W. Wilson, C. C. Van Donkelaar, R. B. van, and R. Huiskes, Journal of Biomechanics, 2005, 38, 1195-1204.

27. C. G. Armstrong, Proceedings of the Institution of Mechanical Engineers: Part H, Journal of Engineering in Medicine, 1986, 15, 55-61.

28. A. Oloyede, R. Flachsmann, and N. D. Broom, Connective Tissue Research, 1992, 27, 211-214.

29. D. E. Shepherd and B. B. Seedhom, Rheumatology (Oxford), 1999, 38, 124-132.

30. M. K. Barker and B. B. Seedhom, Rheumatology (Oxford), 2001, 40, 274-284.

31. G. E. Kempson, in Adult articular cartilage, ed.M. A. R. Freeman, Pitman Medical, Tunbridge Wells, 2 edn., 1979, pp. 333-414.

32. J. E. Jeffrey and R. M. Aspden, Proceedings of the Institution of Mechanical Engineers: Part H, Journal of Engineering in Medicine, 2006, 220, 677-686.

33. E. L. Radin, I. L. Paul, and M. Lowy, Journal of Bone and Joint Surgery, 1970, 52-A, 444-456.

34. G. R. Higginson and J. E. Snaith, Proceedings of the Institution of Mechanical Engineers: Part H, Journal of Engineering in Medicine, 1979, 8, 11-14.

35. L. V. Burgin and R. M. Aspden, Journal of Material Science: Materials in Medicine, 2008, 19, 703-711.

36. J. J. Garcia, N. J. Altiero, and R. C. Haut, Journal of Biomechanical Engineering, 1998, 120, 608613.

37. R. J. Lewis, A. K. MacFarland, S. Anandavijayan, and R. M. Aspden, Osteoarthritis and Cartilage, 1998, 6, 383-392.

38. L. V. Burgin and R. M. Aspden, Medical Engineering and Physics, 2007, 29, 525-530.

39. W. J. Stronge, in Impact Mechanics, Cambridge University Press, Cambridge, 2000.

40. A. Maroudas, in Methods in cartilage research, ed.A. Maroudas, K. E. Kuettner, Academic Press Ltd., London, 1990, ch. Chapter 53, pp. 211-219.

41. R. M. Aspden, Journal of Material Science: Materials in Medicine, 1990, 1, 100-104. 
42. R. M. Aspden, Journal of Biomechanics, 1990, 23, 623.

43. J. S. Jurvelin, M. D. Buschmann, and E. B. Hunziker, Journal of Biomechanics, 1997, 30, 235241.

44. N. O. Chahine, C. C. Wang, C. T. Hung, and G. A. Ateshian, Journal of Biomechanics, 2004, 37, 1251-1261.

45. P. Kiviranta, J. Rieppo, R. K. Korhonen, P. Julkunen, J. Toyras, and J. S. Jurvelin, Journal of Orthopaedic Research, 2006, 24, 690-699.

46. J. S. Jurvelin, M. D. Buschmann, and E. B. Hunziker, Proceedings of the Institution of Mechanical Engineers: Part H, Journal of Engineering in Medicine, 2003, 217, 215-219.

47. L. Cao, I. Youn, F. Guilak, and L. A. Setton, Journal of Biomechanical Engineering, 2006, 128, 766-771.

48. H. Jin and J. L. Lewis, Journal of Biomechanical Engineering, 2004, 126, 138-145.

49. M. S. Laasanen, J. Töyras, R. K. Korhonen, J. Rieppo, S. Saarakkala, M. T. Nieminen, J. Hirvonen, and J. S. Jurvelin, Biorheology, 2003, 40, 133-140.

50. R. K. Korhonen, M. S. Laasanen, J. Toyras, J. Rieppo, J. Hirvonen, H. J. Helminen, and J. S. Jurvelin, J Biomech., 2002, 35, 903-909.

51. O. Demarteau, L. Pillet, A. Inaebnit, O. Borens, and T. M. Quinn, Osteoarthritis and Cartilage, 2006, 14, 589-596. 
Table 1. Published values of Poisson's ratio measured and calculated for articular cartilage.

Measured values tend to be larger than those values calculated on the assumption of a poroelastic model in which Poisson's ratio is assumed to be for that of the solid matrix and is derived by fitting curves to a constitutive equation. Figures are derived from quasi-static testing, and only one study measures anisotropy, maximum $v_{\max }$ and minimum values $v_{\min }$ and strain dependence as shown.

\begin{tabular}{|c|c|c|c|}
\hline Study & \multicolumn{2}{|r|}{ Tissue } & Value \\
\hline & \multicolumn{2}{|r|}{ Calculated } & $v_{\mathrm{S}}$ \\
\hline Jurvelin et al. ${ }^{43}$ & Bovine & Humeral head & $0.174 \pm 0.106$ \\
\hline \multirow{3}{*}{ Chahine et al. ${ }^{44}$} & Bovine & Humeral head, range & $0.027-0.065$ \\
\hline & & mean & $0.13 \pm 0.07$ \\
\hline & Human & Femoral head & $0.14 \pm 0.09$ \\
\hline \multirow{2}{*}{ Kiviranta et al. ${ }^{45}$} & Human & Metatarso-phalangeal & $0.30 \pm 0.07$ \\
\hline & Human & Patella & $0.15 \pm 0.04$ \\
\hline \multirow[t]{2}{*}{ Jurvelin et al. ${ }^{46}$} & Human & Femoral groove, normal & $0.158 \pm 0.148$ \\
\hline & & tangential & $0.180 \pm 0.046$ \\
\hline \multirow[t]{2}{*}{ Cao et al. ${ }^{47}$} & Mouse & Tibial plateau & 0.2 \\
\hline & \multicolumn{2}{|c|}{ Direct optical measurement } & $v$ \\
\hline \multirow{3}{*}{$\begin{array}{l}\text { Jurvelin et al. }{ }^{43} \\
\text { Jin \& Lewis }{ }^{48}\end{array}$} & Bovine & Humeral head & $0.185 \pm 0.065$ \\
\hline & Bovine & Patella, instantaneous & $0.503 \pm 0.028$ \\
\hline & & Patella, equilibrium & $0.463 \pm 0.073$ \\
\hline \multirow{5}{*}{ Laasanen et al. ${ }^{49}$} & Bovine & Patella & $0.24 \pm 0.09$ \\
\hline & & Tibia & $0.38 \pm 0.08$ \\
\hline & & Medial femoral condyle & $0.30 \pm 0.1$ \\
\hline & & Medial patella groove & $0.21 \pm 0.05$ \\
\hline & & Lateral patella groove & $0.19 \pm 0.04$ \\
\hline \multirow[t]{3}{*}{ Korhonen et al. ${ }^{50}$} & Bovine & Humeral head & $0.15 \pm 0.06$ \\
\hline & & Patella & $0.16 \pm 0.05$ \\
\hline & & Medial femoral condyle & $0.21 \pm 0.05$ \\
\hline \multirow[t]{9}{*}{ Demarteau et al. ${ }^{51}$} & Bovine & Humeral head, & \\
\hline & & $20 \%$ strain, $v_{\min }$ & $0.075 \pm 0.051$ \\
\hline & & $20 \%$ strain, $v_{\max }$ & $0.17 \pm 0.05$ \\
\hline & & $40 \%$ strain, $v_{\min }$ & $0.10 \pm 0.06$ \\
\hline & & $40 \%$ strain, $v_{\max }$ & $0.17 \pm 0.06$ \\
\hline & Human & Femoral head & $0059+0037$ \\
\hline & & $20 \%$ strain, $v_{\max }$ & $0.17 \pm 0.05$ \\
\hline & & $40 \%$ strain, $v_{\min }$ & $0.077 \pm 0.034$ \\
\hline & & $40 \%$ strain, $v_{\max }$ & $0.19 \pm 0.06$ \\
\hline
\end{tabular}


Table 2. Mean values (standard deviation) of the maximum longitudinal strain, $\varepsilon_{z}$, calculated from the accelerometer, compared with that measured from the images, the corresponding maximum radial strain, $\varepsilon_{r}$, and Poisson's ratio, $v$, at maximum strain. The strain rate is calculated from the maximum strain divided by the time taken to achieve that strain. Data are from 3 samples from each of two femoral heads, except one sample was lost due to triggering problems from each femoral head subjected to a $75 \mathrm{~mm}$ drop.

\begin{tabular}{|c|c|c|c|c|c|c|}
\hline \multirow{2}{*}{$\begin{array}{l}\text { Drop } \\
\text { height }\end{array}$} & \multirow{2}{*}{$\begin{array}{c}\text { Femoral } \\
\text { head }\end{array}$} & \multirow{2}{*}{$\begin{array}{c}\begin{array}{c}\text { Instrumented } \\
\text { drop tower }\end{array} \\
\varepsilon_{z}\end{array}$} & \multicolumn{3}{|c|}{ Image analysis } & \multirow{2}{*}{$\underset{\left(\mathrm{s}^{-1}\right)}{\text { Strain rate }}$} \\
\hline & & & $\varepsilon_{z}$ & $\varepsilon_{r}$ & $v$ & \\
\hline $25 \mathrm{~mm}$ & $\mathrm{~A}$ & $-0.471(0.028)$ & $-0.475(0.031)$ & $0.41(0.15)$ & $0.86(0.28)$ & $222(11)$ \\
\hline $25 \mathrm{~mm}$ & B & $-0.421(0.020)$ & $-0.376(0.095)$ & $0.235(0.094)$ & $0.63(0.19)$ & $322(7)$ \\
\hline $50 \mathrm{~mm}$ & $\mathrm{~A}$ & $-0.625(0.016)$ & $-0.637(0.015)$ & $0.606(0.040)$ & $0.95(0.05)$ & $339(66)$ \\
\hline $50 \mathrm{~mm}$ & B & $-0.527(0.072)$ & $-0.475(0.046)$ & $0.24(0.13)$ & $0.49(0.22)$ & $458(35)$ \\
\hline $75 \mathrm{~mm}$ & A & $-0.691(0.003)$ & $-0.681(0.050)$ & $0.640(0.033)$ & $0.94(0.02)$ & $418(81)$ \\
\hline $75 \mathrm{~mm}$ & B & $-0.558(0.022)$ & $-0.521(0.015)$ & $0.53(0.10)$ & $1.03(0.22)$ & $527(20)$ \\
\hline
\end{tabular}




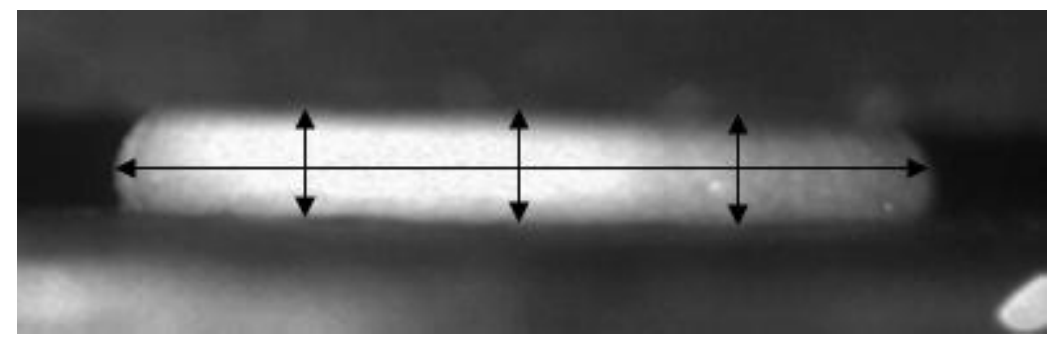

Figure 1. Dimensions of each cartilage sample were measured as shown above in each frame following calibration of the camera; the thickness was the mean of three measurements, the width was taken across the widest point of the sample.

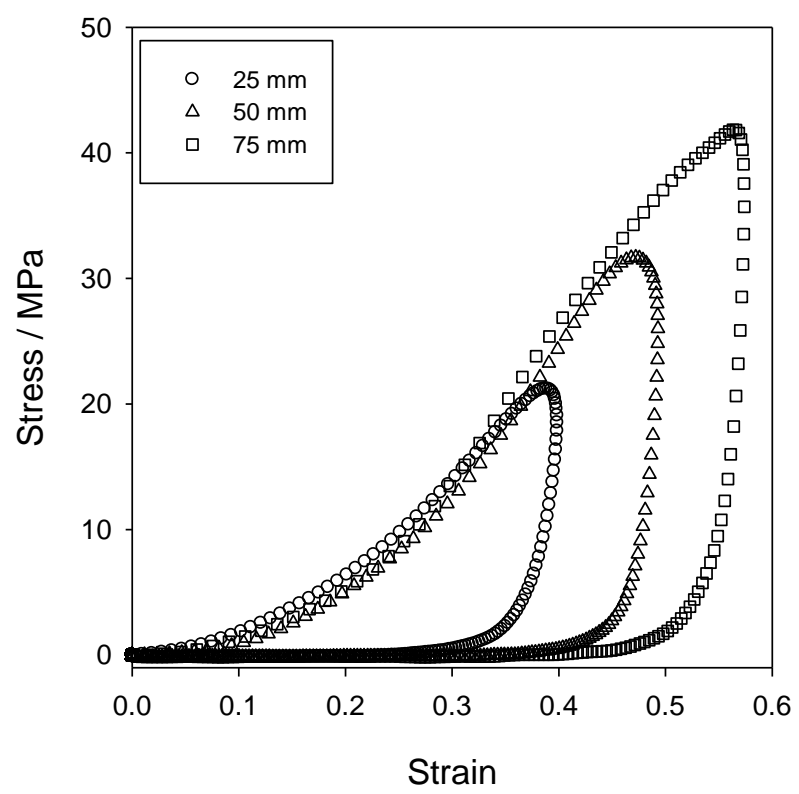

Figure 2. Typical stress-strain curves for a $0.5 \mathrm{~kg}$ mass dropped from 25,50 and $75 \mathrm{~mm}$ onto samples from one individual showing the dependency on impact velocity. Unloading is represented by the unconstrained rebound. 

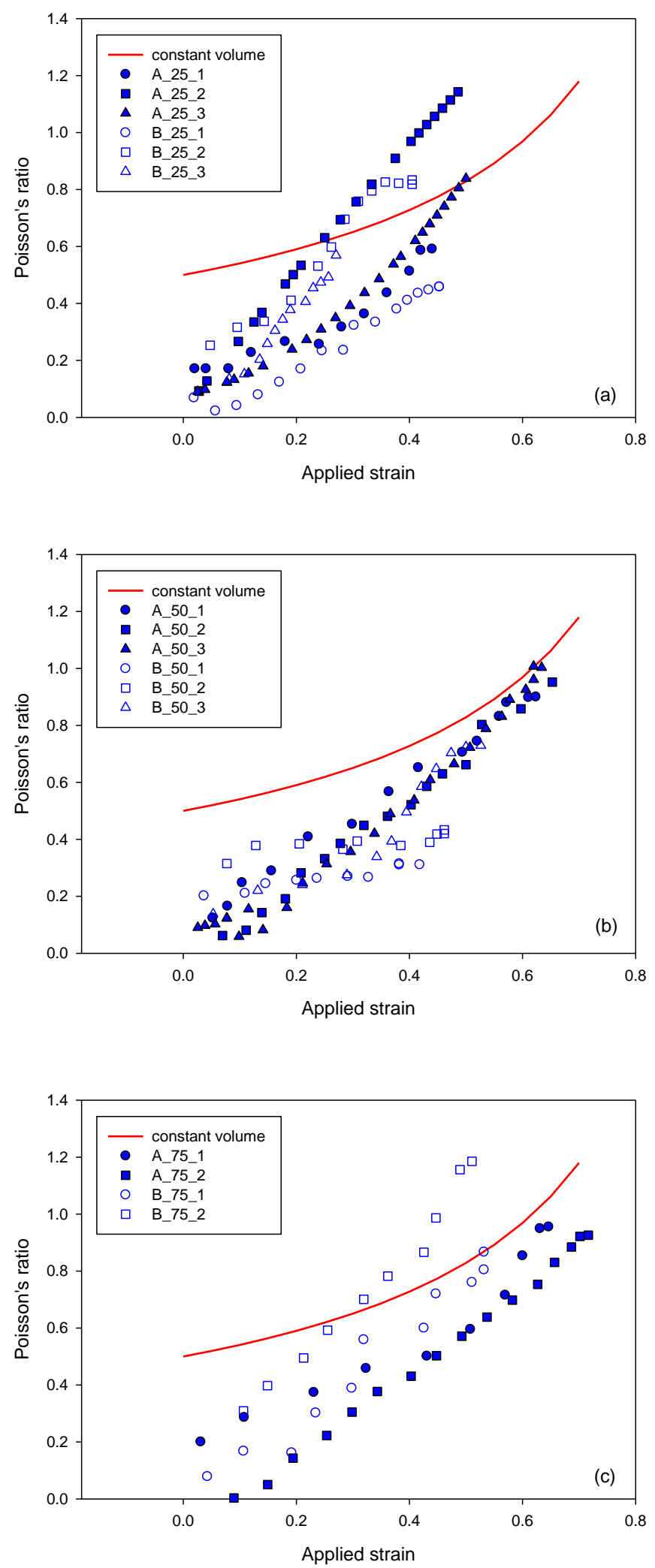

Figure 3. Plots of Poisson's ratio as a function of longitudinal strain from each femoral head, A and $\mathrm{B}$, for drop heights of (a) $25 \mathrm{~mm}$, (b) $50 \mathrm{~mm}$ and (c) $75 \mathrm{~mm}$. Compressive strain traditionally takes a negative sign but is shown positive here for convenience. Also shown as a solid line is a plot of the theoretical ratio calculated for an isovolumetric deformation of a homogeneous, isotropic material. 


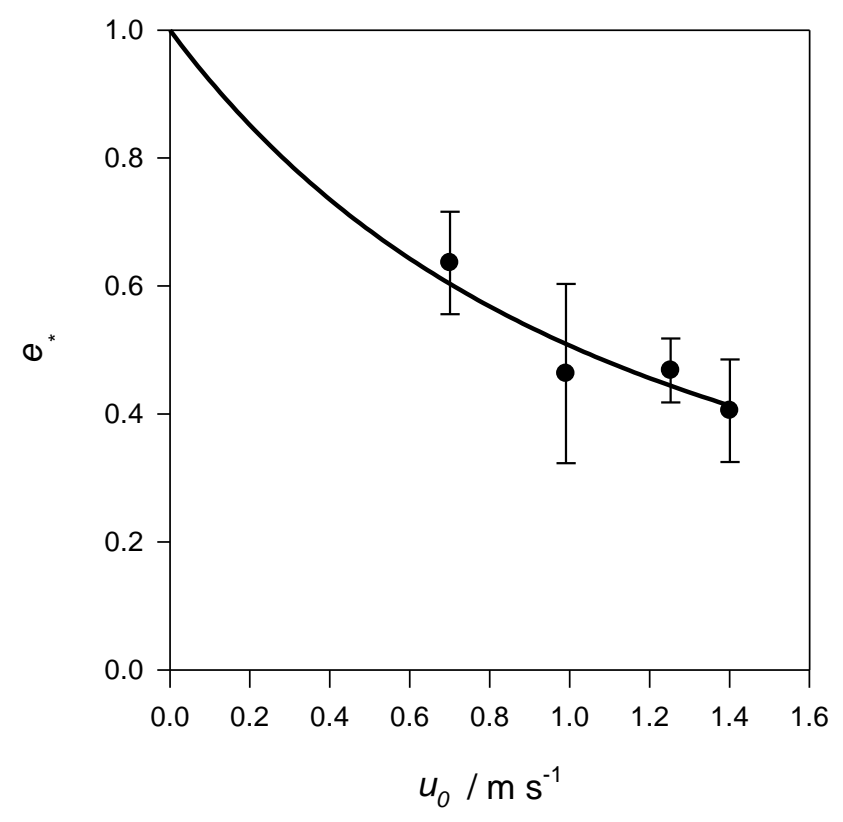

Figure 4. A non-linear viscoelastic element predicts a coefficient of restitution that decreases with increasing impact velocity. The data points are from 4 drop heights for a $0.1 \mathrm{~kg}$ impactor onto bovine articular cartilage and the curve is that fitted from equation 4 with $c / k=1.402$ (standard error $0.092, R^{2}=0.87$ ).

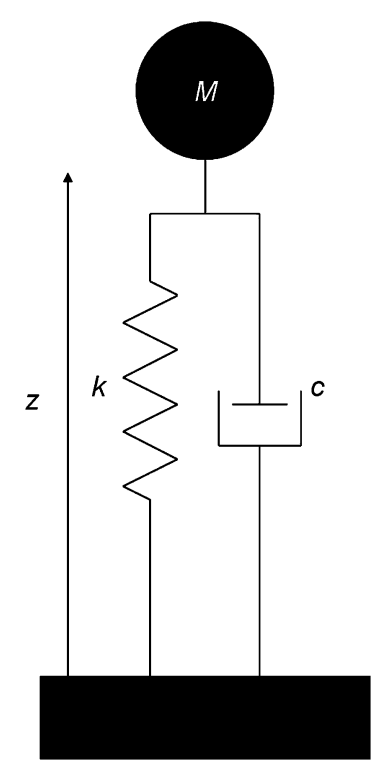

Figure 5. Impact of a mass, $M$, onto a sample represented by a non-linear Kelvin-Voigt viscoelastic element comprising a spring, with spring constant $k$, in parallel with a dashpot, in which the damping coefficient depends on displacement, using a damping coefficient of $c$. 